\title{
The Tammes Problem
}

\section{El Problema de Tammes}

\author{
Enrique Antoniano \\ Universidad Anáhuac México
}

\begin{abstract}
We submit an outline for the solved cases of Tammes problem. We give an elementary proof that the Platonic polihedra; tetrahedom, octaedronm and icosahedrom are the solutions for 4,5 and 12 respectively, based on the determination of the triangles with minmun area. We higlight the concept of star of a vertex, and how the determination of the minimun area stars lead to the solution for the cases 8 and 24 .
\end{abstract}

Keywords. Spherical geometry, triangle, polyhedra, star.

Resumen. Presentamos una reseña de los casos resueltos para el problema de Tammes. Damos una demostración elemental de que los poliedros Platónicos; tetraedro, octaedro e icosaedro son las soluciones para 4, 5 y 12 respectivamente, basada en la determinación de los triángulos con mínima área. Resaltamos el concepto de estrella de un vértice y como la determinación de las estrellas de mínima área conducen a la solución en los casos 8 y 24 .

Palabras Clave. Geometría esférica, triángulo, poliedro, estrella.

Como citar. Enrique Antoniano, "The Tammes Problem", Jou. Cie. Ing., vol. 11, no. 1, pp. 72-82, 2019. doi: $10.46571 / \mathrm{JCl} .2019 .1 .8$

\section{Introduction}

In 1930 the Dutch botanist R.M.L. Tammes [9] noticed that certain arrangements of pollen grains were favored. The pollen grains can be considered as nonoverlapping circular caps on the surface where they grow, supposedly spherical. Tammes conjectured that the arrangements maximized the number of grains considering its diameter and the radius of the spherical surface where they grow.

We can translate this problem to geometry as follows: Place on the surface of the unit sphere the largest number of circles of a given diameter.

The problem is preceded by the famous discussion between Isaac Newton and David Gregory in 1694 [10] about the greatest number of billiard balls that can be placed around the black ball, touching it. Newton said that only 12 when Gregory argued that they could be 13 . Newton was right.

If the reader thinks a bit, the problem can be rephrased as follows:

"Place n points on the surface of the sphere of radius 1 so that the two closest ones are as distant as possible."

This is the formulation that we will have in mind going forward. 


\section{Notation and some considerations about the solutions}

We denote by $S^{2}$ the surface of the unit sphere in the Euclidean space $R^{3}$. If $\wp \subset S^{2}$ is a subset of $n$ points, we write $\#(\wp)=n$ and say that $\wp$ is a positioning of $n$ points on the sphere.

If a segment between two points on the sphere measures $a$, its radial projection is a geodesic arc on the sphere whose length is $\alpha$, the angle that subtends from the center, measured in radians. It is not difficult to see that $\alpha=2 \sin \frac{\alpha}{2}$ or $\alpha=\cos ^{-1} 1-\frac{\alpha^{2}}{2}$, so each determines the other. As $0<\frac{\alpha}{2}<\frac{\pi}{2}$ and since in that range the sin function is increasing, we can use a or $\alpha$ indistinctly in the formulation of the Tammes problem. We will use $\alpha$.

Given a positioning $\wp$ we denote by $\alpha(\wp)$ the minimum distance between two any of its points and by $\alpha(n)=\operatorname{Max}\{\alpha(\wp) \mid(\wp)=n\}$. If $\wp=n$ and $\alpha(\wp)=\alpha(n)$ then the positioning $\wp$ is a solution to the Tammes problem for $n$ points. If in addition $\alpha(\wp \cup\{x\})<\alpha(\wp) \forall x \in \mathrm{S}^{2}$ we say that $\wp$ is an optimal solution. That is, the solution is optimal when we can not add one more point without reducing the minimum distance between pairs of points. Given a positioning $\wp$, we can consider the convex envelope of $\wp$ in $R^{3}$ which is a convex polyhedron. Moreover, the faces of this polyhedron are not only convex, but are also inscribed in a circle. If we divide the non-triangular faces into triangles and project them radially towards the sphere, we obtain a triangulation of the sphere. Using the fact that the Euler characteristic of the sphere is 2 , we have \#(vertices)-\#(edges)+\#faces=2 and then for any triangulation if \#(vertices)=n, then \#(edges $)=3 n-6$ and $\#($ faces $)=2 n-4$.

If $\wp$ is a solution, the edges of the induced triangulation all measure at least $\alpha=\wp$. If in addition $\wp$ is optimal then they are smaller than $2 \alpha$, otherwise the circumcircle of any of the faces adjacent to that edge would have radius greater than or equal to $\alpha$, so that adding to $\wp$ its center would not increase the minimum distance between pairs of points.

Given a spherical triangle with vertices at points $A, B$ and $C$, we will refer to it as $\triangle A B C$ and abusing the language we will denote its angles also by $A, B$ and $C$ and its opposite sides or its measurements by $\alpha, \beta$ and $\gamma$ respectively. In occasions we will denote the angle $A$ as $\nless B A C$ and the side as $\hat{C B}$, that we read arc from $C$ to $B$, to be more precise.

Given the $\triangle A B C$, its cap is the spherical circle that contains the triangle, limited by the circumcircle of it. We denote by cap (ABC) indistinctly that circle or to its spherical radius. Now a theorem [MH, Theorem 4.1], which we will call the spherical central angle theorem:

Spherical Central Angle Theorem: Given $\triangle A B C$, let $O$ be the center of cap $A B C$, then $C-A-B= \pm 2 \nless O A B$ and it will be \pm depending on whether the $\operatorname{arc} A \hat{C} B$ is smaller or larger than a half circumcircle.

Proof: It follows from observing the figure.

This theorem reveals another highly relevant to the problem of Tammes, but before another equally relevant $[\mathrm{MH}]$ :

Girard's formula (1620): The area of $\triangle A B C$ is equal to $A+B+C-\pi$, of

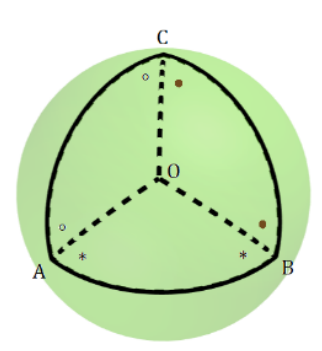
course $A, B$ and $C$ measured in radians. The following theorem is crucial for all the argument that follows. It basically says that given a segment $\hat{A B}$, the locus of the points $C$ for which the triangle $\triangle A B C$ has constant area, is a circle. 
Theorem: Given $\triangle A B C$, let $A^{*}$ and $B^{*}$ be the antipodal points of $A$ and $B$ respectively and $P$ any point in the arc of the circle $A^{*} \hat{C} B^{*}$. Then $\operatorname{Area}(\triangle A B C)=$ $\operatorname{Area}(\triangle A B P)$.

Proof: The triangles $\triangle A B C$ and $\triangle A^{*} B^{*} P$ share the vertex $P$ and since the arcs $A \hat{P} A^{*}$ are geodesic, the angles in $P$ both triangles are equal for being opposed by the vertex. On the other hand $A^{*}=\pi-A$ and y $B^{*}=\pi-B$ since they are internal alternates.

Let $O$ be the center of the arc $A^{*} \hat{C} B^{*}$; by the central angle theorem for $\triangle A^{*} B^{*} P$, we have $P-A^{*}-B^{*}= \pm 2 \not \leq O A^{*} B^{*}$ or $P-(\pi-A)-(\pi-B)=$ $P+A+B-2 \pi=\not \leq O A^{*} B^{*}$ and therefore Area $(\triangle A B P)=P+A+B-\pi=\pi \pm \not \leq O A^{*} B^{*}$ that does not depend on $P$.

In $[\mathrm{K}]$ we can find an excellent and brief treatise on spherical geometry, which I strongly recommend reading, where we can find among others the following law of spherical cosines for $\triangle A B C$ :

$$
\begin{gathered}
\cos (\alpha)=\cos (\beta) \cos (\gamma)+\sin (\beta) \sin (\gamma) \cos (A) \\
\cos (A)=-\cos (B) \cos (C)+\sin (B) \sin (C) \cos \cos (\alpha)
\end{gathered}
$$

By using this Law of Cosines it is not difficult to see that if $A=A(\alpha)$ is the angle of the equilateral triangle of side $\alpha$, then $\cos \cos A=\frac{\cos \cos (\alpha)}{(\alpha)}$ and $\cos \cos \alpha=\frac{\cos \cos (A)}{(A)}$.

Note that as $\alpha>0, \cos \cos \alpha<1$ and $\cos \cos A<\frac{1}{2}$ or $A>\frac{\pi}{3}=60$.

Let us now take $\wp$, an optimal solution to the Tammes problem and let $\alpha=\wp$ and $A=A(\alpha)$. We say that a triangle is admissible if it appears in the triangulation of the sphere induced by such a $\wp$. After the next proposition, we will call a triangle admissible, or $\alpha$ admissible to be more precise, when it satisfies the conditions of the proposition.

Proposition: Let $\beta$ be one side and $B$ an angle of an admissible triangle $T$, then:

a) $\operatorname{cap}(T)<\alpha$

b) $\alpha \leq \beta<2 \alpha$

c) $\frac{A}{2}<B<2 A$

d) At least one of the angles of $T$ is $\geq A$

\section{Proof:}

a) If cap $(T) \geq \alpha$ then we can add to $\wp$ the center of cap $(T)$ and $\wp$ would not be optimal.

b) Necessarily $\alpha \leq \beta$ since $\alpha$ is the minimum distance between two points and on the other hand, as we have already seen, if $\beta \geq \alpha$ then cap $(T) \leq \alpha$, which is impossible.

c) First we observe that when joining two equilateral triangles of side $\alpha$ we form a quadrilateral, that if we split in two it produces an isosceles triangle (red) with an angle equal to $2 A$ and the other two $\frac{A}{2}$. Also, the radius of the cap of this triangle is $\alpha$. 
If a triangle (green) has an angle $B \geq 2 A$, place the red triangle with the vertex of angle $2 A$ over the vertex of the angle in question, as shown in the figure. It is clear by observing the figure that that triangle will have cap $>\alpha$ and therefore it is not admissible.

Moreover, the only triangle with an angle $2 A$, sides $>\alpha$ and cap $\leq \alpha$ is red, which is not admissible. So thencap $B<2 A$.

Finally, we will see that $\frac{A}{2}<B$. For this first we will demonstrate the following:

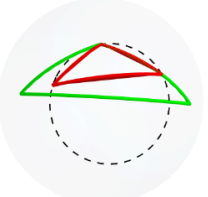

Proposition: If we fix arc $\hat{A B}$ and move the vertex $\hat{A B}$ towards $P$ over the arc $A B C$, then $\nless(C)$ grows. Note that here $P$ is such that $\triangle A P B$ is isosceles.

Proof: By the central angle theorem $C-A-B=c t$, but when moving $C$ in the indicated direction, area $\triangle A B C=A+B+C=2 C-$ cte increases and therefore $C$ increases.

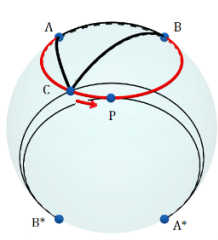

Corollary: If we fix the angle $C$ on a circle and $A$ and $B$ are the intersections of the sides of the angle with that circle, then when the bisector of the angle is rotated as shown in the figure, the length of the arc $\hat{A B}$ decreases. Now, suppose that in the admissible triangle $T, \frac{A}{2} \geq B$.

Let $\hat{B A}$ be the shortest side of the adjacent ones to $B$ and consider the

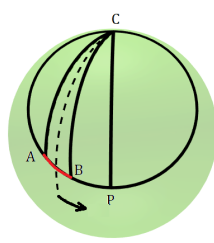
circumference of radius passing through its vertices and leaving the other vertex $C$ of $T$ on the side of its center. Let $P$ be the point of intersection of the circumference and the geodesic throw $\hat{B C}$. Then, by the corollary, $\hat{A P}<\alpha$ and consequently $C$ is outside or on the circle and cap $T \geq \alpha$. Then $\frac{A}{2}<B$.

As a matter of fact, the only triangle with $\operatorname{cap}(T) \leq \alpha$ and an angle $\leq \frac{A}{2}$ is the isosceles with angles $\frac{A}{2}, \frac{A}{2}$ and $A 2$.

d) Let $\triangle X Y Z$ be an admissible triangle and suppose that $X \leq A, Y \leq A$ and let $\gamma \geq \alpha$ be the opposite side to the vertex $Z$.

Then $\sin (X) \sin (Y) \leq \sin ^{2}(A),-\cos (X) \cos (Y) \leq-\cos ^{2}(A) s(\gamma) \leq \cos (\alpha)$ and applying the law of cosines we have

\footnotetext{
$\cos \cos (Z)=-\cos \cos (X) \cos \cos (Y)+\sin (X) \sin (Y) \cos \cos (\gamma) \leq-(A)+\sin ^{2}(A) \cos (\alpha)=$ $-(A)+(1-(A)) \cos \cos \frac{(A)}{1-\cos \cos (A)}=\cos \cos A$, so $Z \geq A$.
} 


\section{The Known solutions}

The solutions are known only for the values of $n \leq 14$ and $n=24$. We will give some values of $a, \alpha$ and $A$, as well as a geometric description of the positioning in each case.

\begin{tabular}{|c|c|c|c|c|c|}
\hline$\#$ & Euclidean distance & Geodesic distance & Angle & Name & Kind \\
\hline$n$ & $a$ & $\alpha$ & $A$ & & \\
\hline 2 & 2 & $\pi$ & $2 \pi$ & Antipodals & Line \\
\hline 3 & 1 & $\frac{2 \pi}{3}$ & $\pi$ & Equilateral & Triangle \\
\hline 4 & $2 \sqrt{\frac{1}{3}}$ & $\cos ^{-1}-\frac{1}{3}$ & $\frac{2 \pi}{3}$ & Tetrahedron & Platonic \\
\hline 5 & $\sqrt{2}$ & $\frac{\pi}{2}$ & $\frac{\pi}{2}$ & & \\
\hline 6 & $\sqrt{2}$ & $\frac{\pi}{2}$ & $\frac{\pi}{2}$ & Octahedron & Platonic \\
\hline 7 & & & $\frac{4 \pi}{9}$ & & \\
\hline 8 & $2 \sqrt{\frac{4-\sqrt{2}}{7}}$ & $\cos ^{-1}-\frac{2 \sqrt{2}-1}{7}$ & $\cos ^{-1}-\frac{\sqrt{2}-1}{2}$ & Quadrangular Antiprism & Antiprism \\
\hline 11 & $\sqrt{\frac{2(5-\sqrt{5})}{5}}$ & $\cos ^{-1}-\frac{1}{\sqrt{5}}$ & $\frac{2 \pi}{5}$ & & \\
\hline 12 & $\sqrt{\frac{2(5-\sqrt{5})}{5}}$ & $\cos ^{-1}-\frac{1}{\sqrt{5}}$ & $\frac{2 \pi}{5}$ & Icosahedron & Platonic \\
\hline 24 & $2 \sqrt{\frac{-3}{-8+(19-3 \sqrt{33})^{\frac{1}{3}}+(19+3 \sqrt{33})^{\frac{1}{3}}}}$ & $\sim 41.4$ & $\sim 65.2$ & Snub Cube & Arquimedian \\
\hline
\end{tabular}

It is extremely interesting to note that according to Robinson [R], Tarsky's general method [T], theoretically provides a solution to the problem of Tammes. In fact, given n, we can obtain an algebraic equation in a finite number of steps that is satisfied by $a(n)$. Therefore, the solution to Tammes's problem is an algebraic number.

\section{Proof of the solutions for $n=2,3,4,6$ and 12}

Theorem: The area of an admissible triangle is less than or equal to the area of the equilateral triangle with sides $\alpha$. Moreover, the inequality is strict unless the admissible triangle is the equilateral one.

Proof: Let $\triangle X Y Z$ be an admissible triangle, and $X$ be the largest of its angles. Then $X \geq A$ since at least one of the angles must meet that condition. If the sides adjacent to $X$ are $>\alpha$ we can cut them to have a triangle of smaller area $\triangle X B C$ with sides adjacent to $X$ equal to $\alpha$. Now we will see that of all the triangles with two sides of length $\alpha$ and its comprehended angle $\geq A$, the one with smaller area is the equilateral. This follows from observing the figure at the right. The dotted circle arcs are of radius $\alpha$ and the black circle arc $A^{*} \hat{C} B^{*}$ is the locus of points $C$ where the triangles $\triangle A C B$ have the same area. Triangles such as $\triangle A P B$ have a larger area since the vertex $P$ falls inside the circumcircle of the arc. Another possibility for $\mathrm{P}$ would be to be at the intersection of the $\operatorname{arc} A^{*} \hat{C} B^{*}$ with the dotted circle centered at $B$, but in that case the triangle is not admissible because it has cap $=\alpha$.

It follows immediately from this theorem, that the solutions for $n=4,6$ and 12 are the Platonic solids; tetrahedron, octahedron and icosahedron, since their faces are equilateral triangles of minimum and just area to fill the sphere. The cases $n=2$ and 3

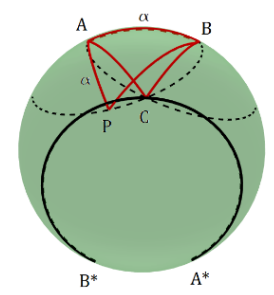
are the same, only that the polyhedra degenerate; one is a diameter (of dimension one) and the other an equilateral triangle (of dimension 2).

This proof is conceptually simpler than any of those found so far in the literature. 


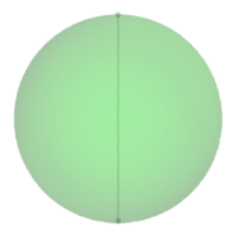

Linea

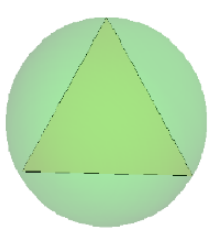

Triángulo

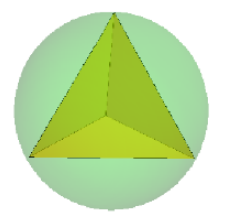

Tetraedro

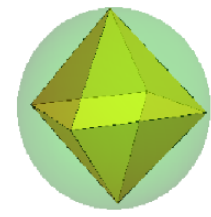

Octaedro

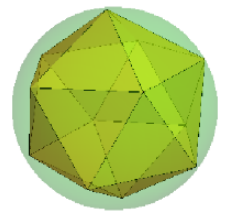

Icosaedro

\section{The cases $n=5$ and 7}

For $n=5$, let $\wp$ be a solution. Necessarily there is a plane that contains three of the points and leaves the other two one on each side. Then necessarily the plane cuts the sphere at an equator (ie a circle of radius 1) and the other two points should be the poles of that equator. Then as the distance of a pole to any point of the equator is $\frac{\pi}{2}$, this is the value of $\alpha(5)=\alpha(6)$.

A non-optimal solution is obtained by eliminating one of the vertices of the octahedron. Any other solution is obtained by moving the three points on the equator by placing them at a distance greater than $\frac{\pi}{2}$ for which there are infinite possibilities. Any of these other solutions is optimal.

For $n=7$, we know a positioning with $A=\frac{4 \pi}{9}$, in which there are two parallel equilateral triangles rotated 60one with respect to the other, one of side $\alpha$ and the other with cap $=\alpha$ being the center of the cap the seventh point. Therefore, in any solution necessarily $A \leq \frac{4 \pi}{9}$.

Let $\wp$ be a solution and take a plane that leaves a point on one side and the rest on the other side. This plane can contain 3 or 4 points, because 5 doesn't fit even at an equator. If the plane has 4 points, rotate it about a line by two consecutive of its points and to the side where the other two are, until you touch one of them. If it touches both, then change the rotation line by moving to the next consecutive points, when doing this operation, the plane will touch only remaining two points. In this way, we get a plane that contains three points let's say $A, B$ and $C$, leave one of say $P$ on one side and the other three say $\mathrm{Q}, \mathrm{R}$ and $\mathrm{S}$ on the other. The radius of the circumcircle of $\triangle A B C$ should be $\alpha$ and $P$ its circumcenter, otherwise the plane that contains them could move parallel to $P$ without increasing the minimum distance, but moving it away from the plane by $Q, R$ and $S$ so when moving also that last plane would increase the minimum distance, contradicting the fact that the positioning was optimal. The triangles $\triangle A B C$ and $\triangle Q R S$ must be equilateral, obtaining the desired solution.

This kind of argument is hopeless for higher values of $n$.

\section{The cases $n=8$ and 24}

It is about a pair of solids, the Quadrangular Antiprism for $n=8$ and the Snub Cube, an Arquimedian for $n=24$.

The Quadrangular Antiprism has 8 triangular and 2 square faces. At each vertex, 3 triangles and 1 square forming identical dihedral angles at each vertex.

The Snub Cube has 32 triangular and 6 square faces. At each vertex, 4 triangles and 1 square forming at identical dihedral angles at each vertex.

The proof that these two polyhedra are optimal solutions lies in

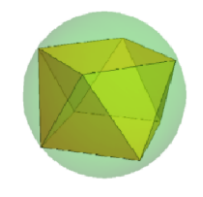

Quadrangular Antiprism

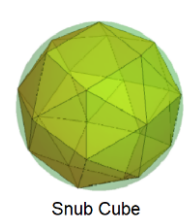
the consideration of the stars of the vertices. I remember that the polyhedron must be projected radially towards the sphere and it must be triangulated. In these two cases the squares must be triangulated, including any of their diagonals.

Definition: The star of a vertex of a polyhedron is the union of the faces that impinge on it. The valence of the vertex is the number of incident faces, or equivalently the number of incident edges. When 
the polyhedron is triangulated, all faces are triangles.

A star is admissible if its faces are admissible and what we want is to determine the minimum area of an admissible star according to its valence. This is what Robinson [R, Theorem Aa] does.

To get into the background of what Robinson does, let $\alpha$ be a given distance. We say that a positioning $\wp$ is saturated if the distance between any pair of its points is and no other point can be added without losing this property.

Let $A$ be the angle of the equilateral triangle of side $\alpha$ and let $\beta$ and $B$ be the diagonal and the angle of the square of side $\alpha$. Denote by $\triangle$ the equilateral triangle of side $\alpha$, by $\triangle^{*}$ the isosceles triangle with two sides $\alpha$ and angle between them $W=2 \pi-4 A$ and by $C$ its other two angles. Abusing the notation, we will also denote by $\triangle$ and $\triangle^{*}$ the areas of said triangles. It is to be noted that for the Snub Cube $W=B$.

Theorem [R, Theorem Aa]: Suppose $60<A<72$. Consider a standard triangulation corresponding to a saturated point system relative to the distance $\alpha$. Then, if the valence of a vertex is $k$, its total area is greater or equal than $4 \triangle+k-4 \triangle^{*}$. Equality holds only when $k=5$ and there are four equilateral triangles of side or when $k=6$, there are four equilateral triangles of side $\alpha, W \geq B$, and the other two triangles together form a rhombus (quadrilateral of equal sides).

The proof of this theorem takes much of Robinson's work [R], where he does it in a very bright and geometrical way. As a corollary we have the solution for $n=24$ as follows.

Theorem [R]: The Snub Cube is the solution to Tammes problem for $n=24$.

Proof: We will deviate a bit from Robinson's argument. Let $e_{\mathrm{k}}$ be the number of stars with valence $k$, then $\sum e_{k}=n$ and y $\sum k e_{k}=6 n-12$ since in the last expression we have added 2 times each edge. Thus the minimum possible sum for the areas of the stars is:

$$
\begin{gathered}
\left.\sum\left(4 \triangle+(k-4) \triangle^{*}\right) e_{\mathrm{k}}=\sum\left(4 \triangle-4 \triangle^{*}\right) e_{\mathrm{k}}+\sum k \triangle^{*}\right) e_{\mathrm{k}}= \\
4 n\left(\triangle-\triangle^{*}\right)+(6 n-12) \triangle^{*}=4 n \triangle+(2 n-12) \triangle^{*}
\end{gathered}
$$

In the case of the Snub Cube, we know that $n=24$ and $32 \triangle+12 \triangle^{*}=4 \pi$, so

$$
4 n \triangle+(2 n-12) \triangle^{*}=96 \triangle+36^{*}=12 \pi
$$

Then $12 \pi=\sum\left(4 \triangle+(k-4) \triangle^{*}\right) e_{\mathrm{k}} \leq \sum$ Area of starts $=12 \pi$ and therefore all stars must be minimal and the valences 5 or 6 . Now, by the law of cosines applied to the triangle $\triangle^{*}$ we have:

$$
\begin{gathered}
\cos \cos \beta=\cos ^{2} \alpha+\sin ^{2} \alpha \\
\cos \cos W=-\cos ^{2} C+\sin ^{2} C \cos \cos \beta
\end{gathered}
$$

Since $W=2 \pi-4 A$ y $\cos \alpha=\cos \cos (A) /(1-\cos A)$ we can see that:

$$
c=\operatorname{Arc} \operatorname{Cos} \sqrt{\frac{4 \cos ^{4} A(1+\cos A)}{2+2 \cos A+2 \cos 2 A+\cos 3 A+\cos 4 A}}
$$


And with this verify that $4 \triangle+(k-4) \triangle^{*}=4(7-k) A+2(k-4) C+(k-8) \pi$ is increasing with A $\forall k$.

Then, for $n=24, A$ and $\alpha$ have to be as in the Snub Cube and this is the unique possible solution.

Let now $\triangle^{\prime}$ be the isosceles triangle with two sides and angle between them $X=2 \pi-3 A$ and let $D$ be its other two angles. As before, we will denote also by $\triangle^{\prime}$ the area of say triangle. It should be noted that in the case of the Quadrangular Antiprism, $X=X=2 D$, and two triangles $\triangle^{\prime}$ stuck together by their major side form a square.

Conjecture: For $\alpha \geq 2 \sqrt{\frac{4-\sqrt{2}}{7}}$ which is the value for the Quadrangular Antiprism, if the valence of a vertex is $\mathrm{k}$, its total area is greater than or equal than $3 \triangle+(k-3) \triangle^{\prime}$. Equality occurs only when $k=4$ and there are three equilateral triangles of side or when $k=5$, there are three equilateral triangles of side and the other two triangles together form a rhombus (quadrilateral of equal sides).

Theorem: The Quadrangular Antiprism is the solution to Tammes problem for $n=8$.

Proof: The proof is completely similar as for the Snub Cube. If $e_{\mathrm{k}}$ is the number of stars of valence $k$, then $\sum e_{k}=n$ and $\sum k e_{k}=6 n-12$ as before. So the minimum posible sum for the areas of stars is:

$$
\begin{gathered}
\left.\sum\left(3 \triangle+(k-3) \triangle^{\prime}\right) e_{k}=\sum\left(3 \triangle-3 \triangle^{\prime}\right) e_{k}+\sum k \triangle^{*}\right) e_{k}= \\
3 n\left(\triangle-\triangle^{\prime}\right)+(6 n-12) \triangle^{\prime}=3 n \triangle+(3 n-12) \triangle^{\prime} .
\end{gathered}
$$

For the Quadrangular Antiprism, $n=8$ and $8 \triangle+4 \triangle^{\prime}=4 \pi$, so

$$
3 n \triangle+(3 n-12) \triangle^{\prime}=3\left(8 \triangle+4 \triangle^{\prime}\right)=12 \pi
$$

Then $12 \pi=\sum\left(3 \triangle+(k-3) \triangle^{\prime}\right) e_{\mathrm{k}} \leq \sum$ Area of stars $=12 \pi$ and therefore all stars must be minimal, and valences 4 or 5 .

\section{Conclusions}

We claim that in order to get a solution, first you have to guess a position and then to prove that it is a solution.

It is natural to think on the Platonic or Arquimedian positions because of its symmetries, but how is it seen, this is not enough to solve the problem. It is plausible to think that what you need is as much equilateral triangles as possible, although apparently this is not enough either, since Michael S. Longuet-Higgins in [7] claims that the Snub Dodecahedron is not the solution for $n=60$.

In view of the work of Tarsky [13] one should try to find an algorithm to produce the solutions and then try to prove that they are.

\section{Aknowledgments}

The author wish to thanks Enrique Zamora and José de Jesús Angel for their participation in the seminar on the Tammes problem that took place during the summer of 2017 in the Engineering Faculty of Universidad Anahuac, and to Gabriel Velasco for his review and suggestions on this writing.

\section{References}

[1] R. Bowen, "On Sums of Valencies in Planar Graphs", Canadian Mathematical Bulletin, vol. 9, no 1, pp. 111-114, 1966.

[2] R. Bowen, "The generation of minimal triangle graphs", Mathematics of Computation, vol. 21, no 98, pp. 248-250, 1967. doi: 10.2307/2004171.

[3] R. Bowen and S. Fisk, "Generation of Triangulations of the Sphere", Mathematics of Computation, vol. 21, no 98, pp. 250-252, 1967.

[4] L. Danzer, "Finite point-sets on $S 2$ with minimum distance as large as possible", Discrete mathematics, vol. 60, pp. 3-66, 1986. 
[5] G. J. Flores, "El problema de las 13 esferas Parte I y II ”, Facultad de Matemática, Astronomía y Física, Universidad Nacional de Córdoba.

[6] K. Ck. Ikastegia "Trigonometría esférica", 2001. [En línea]. Disponible en: http://www.euskalnet.net/pasku/trigono.pdf. [Accedido: 10-junio-2017].

[7] M. S. Longuet-Higgins, "Snub polyhedra and organic growth", Proc, R. Soc. A., vol. 465, pp. 477-491, 2008.

[8] H. Maehara, "The problem of thirteen spheres - a proof for undergraduates", European Journal of Combinatorics, vol. 28, pp. 1770-1778, 2007.

[9] E. Mooers, “Tammes's problem”, University of Vermont, 1994. [En línea]. Disponible en: http://www.uvm.edu/pdodds/files/papers/others/1994/mooers1994a.pdf. [Accedido: 22-junio-2017].

[10] O. R. Musin, A. S. Tarsov, "The Strong thirteen spheres problem”, Discrete Comput Geom, vol. 48, no. 1, pp. 128-141, 2012. doi: 10.1007/s00454-011-9392-2.

[11] O. R. Musin, A. S. Tarsov, "The Tammes Problem for N = 14" Strong thirteen spheres problem",Experimental Mathematics, vol. 24, no. 4, pp. 460-468, 2015. doi: 10.1080/10586458.2015.1022842.

[12] R. M. Robinson, "Arrangement of 24 points on a Sphere", Math. Annalen, vol. 144, pp. 17-48, 1961. doi: 10.1007/BF01396539.

[13] A. Tarsky, A decision method for Elementary algebra and geometry. Berkeley, California: University of California Press, 1951.

[14] W. P Thurston, "Shapes of polyhedra and triangulations of the sphere" en Geometry Topology Monographs, vol. 1 The Epstein birthday schrift, I. Rivin, C. Rourke y C. Series, England: Mathematica Sciences Publisher, 1998, pp. 511-549. doi: 10.2140/gtm.1998.1.511.
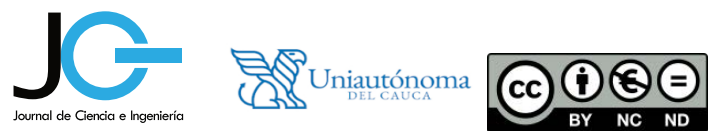